\title{
SMOOTHNESS OF CAUCHY RIEMANN MAPS FOR A CLASS OF REAL HYPERSURFACES
}

\author{
HeRvé Gaussier
}

\begin{abstract}
We study the regularity problem for Cauchy Riemann maps between hypersurfaces in $\mathbb{C}^{n}$. We prove that a continuous Cauchy Riemann map between two smooth $\mathcal{C}^{\infty}$ pseudoconvex decoupled hypersurfaces of finite D'Angelo type is of class $\mathcal{C}^{\infty}$.
\end{abstract}

\section{Introduction}

Many classical problems in complex analysis rely on the boundary behavior of holomorphic maps and, as a consequence, on the regularity of Cauchy Riemann maps between real hypersurfaces. Only partial results have been obtained when the hypersurfaces are not assumed real analytic: the smoothness of a continuous Cauchy Riemann (CR) map between smooth real hypersurfaces was proved, for instance, in $[\mathbf{1 7}]$ for strictly pseudoconvex hypersurfaces, in [11] for pseudoconvex hypersurfaces of finite D'Angelo type in $\mathbb{C}^{2}$, and in [8] for a Lipschitz CR map between convex hypersurfaces of finite D'Angelo type. It is natural to study decoupled hypersurfaces (or domains) to understand the link between complex dimension two and higher complex dimensions: for such domains J. D. McNeal [16] gave estimates on Bergman, Caratheodory and Kobayashi metrics, J. E. Fornæss and J. D. McNeal [14] constructed local peak holomorphic functions at boundary points, and D. C. Chang and S. Grellier [7] gave properties of the Szegö projection under an additive global assumption.

In this paper we prove the following local result:

Theorem 1. Let $\Gamma_{1}$ and $\Gamma_{2}$ be two $\mathcal{C}^{\infty}$ pseudoconvex real hypersurfaces in $\mathbb{C}^{n+1}$, containing the origin 0 , and let $f$ be a continuous non constant CR map from $\Gamma_{1}$ to $\Gamma_{2}$, satisfying $f(0)=0$. If $\Gamma_{1}$ and $\Gamma_{2}$ are decoupled, of finite D'Angelo type at the origin, then $f$ is a smooth $\mathcal{C}^{\infty}$ locally finite map near the origin.

2000 Mathematics Subject Classification. Primary: 32H40, 32H99; Secondary: 32F40, 32G07, 32H15, 32H35, 32M99. 
According to a result of S. Bell and D. Catlin [4], it is sufficient to prove that the set $f^{-1}(f(0))$ is compact near the origin i.e. that for any neighborhood $U$ of 0 the set $f^{-1}(f(0)) \cap U$ is relatively compact in $U$. Using a dilation we reduce the study of the regularity of $f$ to the study of the boundary behavior of a holomorphic map $F$ between rigid algebraic domains $D_{1}$ and $D_{2}$. This map is the limit of some holomorphic maps $F^{\nu}$. The algebraicity of domains $D_{1}$ and $D_{2}$ gives some information on the map $F$, detailed in Proposition 1.2. Moreover one can prove that if the dilated maps $F^{\nu}$ satisfy uniform Hölder estimates then the non compactness of the set $f^{-1}(f(0))$ implies the non compactness of $F^{-1}(F(0))$ (the origin is mapped to the origin by the scaling process applied to $\left.\Gamma_{1}\right)$. Such Hölder estimates may be obtained when the sequence $\left(F^{\nu}(0)\right)_{\nu}$ is bounded; we prove this property on $\left(F^{\nu}(0)\right)_{\nu}$, and consequently Theorem 1 , as soon as the hypersurface $\partial D_{1}$ is not spherical, i.e. not locally biholomorphic to a sphere at a strictly pseudoconvex point. This relies on a classification of vector fields tangent to a weighted homogeneous rigid polynomial hypersurface, given by E. Bedford and S. Pinchuk [3], and on a precise study of the restrictions of $F$ to some subvarieties of $D_{1}$ (Sections 2 and 3). If $\partial D_{1}$ is spherical it may happen that $\lim _{z \in D_{1}}|F(z)|=\infty$. Using the characterization of spherical rigid hypersurfaces, given by A. V. Isaev [15] and obtained in the spirit of the Chern-Moser theory, we describe $F$ in terms of a correspondence of the unit ball. This proves the compactness of $F^{-1}(\infty)$ in $\partial D_{1}$ and ends the proof of Theorem 1 (Section 4). We note that these techniques also provide a modified proof of Theorem 0.1 in [11].

\section{Reduction of the problem}

Decoupled hypersurfaces are defined as follows:

Definition 1.1. A hypersurface $\Gamma$ in $\mathbb{C}^{n+1}$ containing the origin 0 is decoupled at 0 if there are a neighborhood $U$ of 0 , holomorphic coordinates $\left(z_{0}, z\right)=\left(z_{0}, z_{1}, \ldots, z_{n}\right)$ centered at 0 and a defining function $r$ such that:

$$
\Gamma=\left\{\left(z_{0}, z\right) \in U: r\left(z_{0}, z\right)=\operatorname{Im} z_{0}+\sum_{j=1}^{n} f_{j}\left(z_{j}, \bar{z}_{j}\right)=0\right\}
$$

where $f_{j}$ is a real function for every $j=1, \ldots, n$. 
Let $\Gamma_{1}=\left\{\left(z_{0}, z\right) \in U: r_{1}\left(z_{0}, z\right)=0\right\}$ and $\Gamma_{2}=\left\{\left(z_{0}, z\right) \in U\right.$ : $\left.r_{2}\left(z_{0}, z\right)=0\right\}$ be two smooth $\mathcal{C}^{\infty}$ decoupled pseudoconvex hypersurfaces in $\mathbb{C}^{n+1}$, of finite type $2 m$ and $2 k$ respectively (in the sense of D'Angelo [12]) at the origin. We may assume that the functions $f_{j}^{1}$ and $f_{j}^{2}$, in the expansions of $r_{1}$ and $r_{2}$, are subharmonic functions of class $\mathcal{C}^{\infty}$, without harmonic terms, vanishing at order less than or equal to $2 m$ and $2 k$ respectively. If $f$ is a continuous CR map from $\Gamma_{1}$ to $\Gamma_{2}$ then according to $[4]$ we have:

(i) $f$ extends locally to a holomorphic map (still called $f$ ) from the pseudoconvex side $\Omega_{1}$ of $\Gamma_{1}$ to the pseudoconvex side $\Omega_{2}$ of $\Gamma_{2}$,

(ii) the extension $f$ is continuous up to $\Gamma_{1}$ with $f\left(\Gamma_{1}\right) \subset \Gamma_{2}$.

Since the order of contact between the $(j+1)$ th coordinate complex line and $\Gamma_{1}$ at the origin is less than or equal to $2 m$ we may write for every $1 \leq j \leq n$ : $f_{j}^{1}\left(z_{j}, \overline{z_{j}}\right)=H_{j}\left(z_{j}, \overline{z_{j}}\right)+R_{j}\left(z_{j}, \overline{z_{j}}\right)$ where $H_{j}$ is a homogeneous polynomial of degree $2 m_{j} \leq 2 m$ and $R_{j}$ denotes terms of larger degree. If $\left(p^{\nu}\right)_{\nu}$ is a sequence of points in $\Omega_{1}$ converging to 0 then $\left(f\left(p^{\nu}\right)\right)_{\nu}=\left(q^{\nu}\right)_{\nu}$ converges to 0 by (ii). We may assume that there is a unique point $z^{\nu} \in \Gamma_{2}$ such that $\operatorname{dist}\left(q^{\nu}, \Gamma_{2}\right)=\left|q^{\nu}-z^{\nu}\right|=\delta^{\nu}$. Let $L_{0}, \ldots, L_{n}$ be the vector fields defined by $L_{0}=\frac{\partial}{\partial z_{0}}$ and for $1 \leq j \leq n$, $L_{j}=\frac{1}{2 i} \frac{\partial}{\partial z_{j}}-\frac{\partial r_{2}}{\partial z_{j}} \frac{\partial}{\partial z_{0}}$. We note that $\left\{L_{0}-\overline{L_{0}}, L_{1}, \ldots, L_{n}, \overline{L_{1}}, \ldots, \overline{L_{n}}\right\}$ span the real tangent space to $\Gamma_{2}$ and $\left\{L_{1}, \ldots, L_{n}\right\}$ span $\mathbb{C} T^{1,0}\left(\Gamma_{2}\right)$. For every $\nu$, let $\mathcal{L}_{s, t}^{j} r_{2}\left(z^{\nu}\right)$ be the commutator of $L_{j}, \overline{L_{j}}$ of length $s-1$ in $z_{j}$ and $t-1$ in $\overline{z_{j}}$ at $z^{\nu}$. Since $\Omega_{2}$ is of finite type we can set:

$$
\begin{aligned}
& M_{j}=\inf \left\{m_{j} \text { such that } \mathcal{L}_{s, t}^{j} r_{2}\left(z^{\nu}\right) \neq 0\right. \\
& \left.\qquad \text { for some } s, t \text { such that } s+t=m_{j}\right\},
\end{aligned}
$$

$$
\begin{aligned}
C_{l}^{j}\left(z^{\nu}\right) & =\sup \left\{\left|\mathcal{L}_{s, t}^{j} r_{2}\left(z^{\nu}\right)\right|: s+t=l\right\}, \\
\tau_{j}^{\nu} & =\inf \left\{\left(\delta^{\nu} / C_{l}^{j}\left(z^{\nu}\right)\right)^{1 / l}: 2 \leq l \leq M_{j}\right\}
\end{aligned}
$$

and define the dilation:

$$
\Lambda_{2}^{\nu}\left(z_{0}, z\right)=\left(z_{0} / \delta^{\nu}, z_{1} / \tau_{1}^{\nu}, \ldots, z_{n} / \tau_{n}^{\nu}\right)
$$

Let us consider automorphisms $U^{\nu}$ of $\mathbb{C}^{n+1}$ converging to the identity with $U^{\nu}\left(q^{\nu}\right)=\left(-\delta^{\nu}, 0\right)$. We may choose $U^{\nu}$ such that $r_{2} \circ\left(U^{\nu}\right)^{-1}$ is decoupled and has no harmonic terms in $z_{1}, \ldots, z_{n}$. 
If $p^{\nu}=\left(-\varepsilon_{\nu}, 0\right)$ is on the real inward normal to $\Omega_{1}$ at the origin and $\Lambda_{1}^{\nu}$ is the dilation $\Lambda_{1}^{\nu}\left(z_{0}, z\right)=\left(\left(\varepsilon_{\nu}\right)^{-1} z_{0},\left(\varepsilon_{\nu}\right)^{-1 / 2 m_{1}} z_{1}, \ldots,\left(\varepsilon_{\nu}\right)^{-1 / 2 m_{n}} z_{n}\right)$ we may consider the family of maps $F^{\nu}=\Lambda_{2}^{\nu} \circ U^{\nu} \circ f \circ\left(\Lambda_{1}^{\nu}\right)^{-1}$. Without any restriction we may assume that each map $F^{\nu}$ is defined on $\Omega_{1}^{\nu}$ with values in $\Omega_{2}^{\nu}$ where $\Omega_{1}^{\nu}=\left\{\left(z_{0}, z\right) \in \Lambda_{1}^{\nu}(U): r_{1}\left(\left(\Lambda_{1}^{\nu}\right)^{-1}\left(z_{0}, z\right)\right)<0\right\}$ and $\Omega_{2}^{\nu}=\left\{\left(z_{0}, z\right) \in \Lambda_{2}^{\nu}(U): r_{2}^{\nu}\left(z_{0}, z\right)=r_{2}\left(\left(U^{\nu}\right)^{-1} \circ\left(\Lambda_{2}^{\nu}\right)^{-1}\left(z_{0}, z\right)\right)<0\right\}$.

We obtain after extraction of subsequences:

\section{Proposition 1.1.}

(i) The sequence $\left(\Omega_{1}^{\nu}\right)_{\nu}$ converges, in the Hausdorff convergence, to the domain $D_{1}=\left\{\left(z_{0}, z\right) \in \mathbb{C}^{n+1}: \operatorname{Im} z_{0}+\sum_{j=1}^{n} H_{j}\left(z_{j}, \bar{z}_{j}\right)<0\right\}$.

(ii) The sequence $\left(\Omega_{2}^{\nu}\right)_{\nu}$ converges to $D_{2}=\left\{\left(z_{0}, z\right) \in \mathbb{C}^{n+1}: \operatorname{Im} z_{0}+\right.$ $\left.\sum_{j=1}^{n} P_{j}\left(z_{j}, \bar{z}_{j}\right)<0\right\}$ where $P_{j}$ is a non harmonic subharmonic polynomial of degree less than or equal to $2 k$.

(iii) The family $\left(F^{\nu}\right)_{\nu}$ converges uniformly on compact subsets of $D_{1}$ to a holomorphic map $F$ from $D_{1}$ to $D_{2}$.

Proof of Proposition 1.1: The expression of the dilation $\Lambda_{1}^{\nu}$ gives part (i).

Part (ii): by the definition of $\tau_{j}^{\nu}$ there is a real positive constant $C$ such that for every sufficiently large $\nu$ and every $j \geq 2, \tau_{j}^{\nu} \lesssim\left(\delta^{\nu}\right)^{1 / 2 k}$; the sequence $\left(r_{2}\left(\left(U^{\nu}\right)^{-1} \circ\left(\Lambda_{2}^{\nu}\right)^{-1}\right)\right)_{\nu}$ converges to a function $\left(z_{0}, z\right) \mapsto$ $\operatorname{Im} z_{0}+\sum_{j=1}^{n} P_{j}\left(z_{j}, \overline{z_{j}}\right)$ where $P_{j}$ is a subharmonic polynomial of degree less than or equal to $2 k$, without harmonic term.

Part (iii) was proved by F. Berteloot [5] in $\mathbb{C}^{2}$ : let $\left(h^{k}=\left(h_{0}^{k}, \ldots, h_{n}^{k}\right)\right)_{k}$ be a sequence of holomorphic maps from the unit disc $\Delta$ to $\Omega_{2}^{\nu}$ such that $\left(h^{k}(0)\right)_{k}$ is relatively compact in $D_{2}$. According to the Gauss formula we have for every fixed $1 \leq j \leq n$ :

$$
\int_{0}^{1} \frac{d t}{t} \int_{\Delta_{t}} \frac{\partial^{2}\left(r_{2}^{\nu} \circ \tilde{h}^{k}\right)}{\partial z_{j} \partial \bar{z}_{j}}=\frac{1}{2 \pi} \int_{0}^{2 \pi} r_{2}^{\nu} \circ \tilde{h}^{k}\left(e^{i \theta}\right) d \theta-r_{2}^{\nu} \circ \tilde{h}^{k}(0)
$$

where $\tilde{h}^{k}=\left(h_{1}^{k}, \ldots, h_{n}^{k}\right)$ and $\Delta_{t}=\{\lambda \in \mathbb{C}:|\lambda|<t\}$.

Using the expression of $r_{2}^{\nu}$ we get:

$$
\int_{0}^{1} \frac{d t}{t} \int_{\Delta_{t}} \frac{\partial^{2}\left(r_{2}^{\nu} \circ \tilde{h}^{k}\right)}{\partial z_{j} \partial \bar{z}_{j}}=\int_{0}^{1} \frac{d t}{t} \int_{\Delta_{t}} \frac{\partial^{2}\left(\left(r_{2}^{\nu}\right)_{j} \circ h_{j}^{k}\right)}{\partial z_{j} \partial \bar{z}_{j}}
$$

where $\left(r_{2}^{\nu}\right)_{j}$ is the restriction of $r_{2}^{\nu}$ to the $j$ th complex coordinate axis.

However for every $0<r<1$ we have:

$\int_{0}^{1} \frac{d t}{t} \int_{\Delta_{t}} \frac{\partial^{2}\left(\left(r_{2}^{\nu}\right)_{j} \circ h_{j}^{k}\right)}{\partial z_{j} \partial \bar{z}_{j}} \geq \int_{r}^{1} \int_{\Delta_{r}} \frac{\partial^{2}\left(\left(r_{2}^{\nu}\right)_{j} \circ h_{j}^{k}\right)}{\partial z_{j} \partial \bar{z}_{j}} \geq \int_{h_{j}^{k}\left(\Delta_{r}\right)} \frac{\partial^{2}\left(r_{2}^{\nu}\right)_{j}}{\partial z_{j} \partial \bar{z}_{j}}$. 
Hence, since $r_{2}^{\nu} \circ \tilde{h}^{k}\left(e^{i \theta}\right) \leq-\operatorname{Re} h_{0}^{k}\left(e^{i \theta}\right)$, we obtain:

$$
\int_{h_{j}^{k}\left(\Delta_{r}\right)} \frac{\partial^{2}\left(r_{2}^{\nu}\right)_{j}}{\partial z_{j} \partial \bar{z}_{j}} \leq-\operatorname{Re} h_{0}^{k}(0)-r_{2}^{\nu} \circ \tilde{h}^{k}(0) .
$$

The term - Re $h_{0}^{k}(0)-r_{2}^{\nu} \circ \tilde{h}^{k}(0)$ being bounded from above independently of $k$, the sequence $\left(h_{j}^{k}\right)_{k}$ converges uniformly on compact subsets of $\Delta$ to a holomorphic map $h$ from $\Delta$ to $\overline{D_{2}}$. Since $\left(h^{k}(0)\right)_{k}$ is relatively compact in $D_{2}$ we have the inclusion $h(\Delta) \subset D_{2}$. By covering the unit ball of $\mathbb{C}^{n+1}$ by disks we get the same convergence for any sequence $\left(h^{k}\right)_{k}$ of holomorphic maps defined on the unit ball and also for any sequence of holomorphic maps defined on $D_{1}$. This proves part (iii).

It happens that the map $F$ has some intrinsic properties coming mainly from the rigidity of the domains $D_{1}$ and $D_{2}$. For instance we know by [18] that if, in our situation, there is a sequence $\left(z^{\nu}\right)_{\nu}$ of points in $D_{1}$ converging to a point $z^{\infty}$ in $\partial D_{1}$ such that the sequence $\left(F\left(z^{\nu}\right)\right)_{\nu}$ converges to a point $w^{\infty}$ in $\partial D_{2}$ then $F$ extends continuously to a neighborhood of $z^{\infty}$ in $\overline{D_{1}}$ and we can set $F\left(z^{\infty}\right)=w^{\infty}$. In that case we say that $F\left(z^{\infty}\right)$ is a finite point in $\partial D_{2}$.

\section{Proposition 1.2.}

(i) The map $F$ is locally proper i.e. $F$ is proper from $D_{1} \cap B(0, r)$ to $F\left(D_{1} \cap B(0, r)\right)$ for every real positive number $r$.

(ii) If $F(0)$ is a finite point in $\partial D_{2}$ then the set $F^{-1}(F(0))$ is finite near 0 in $\partial D_{1}$.

(iii) There exist strictly pseudoconvex points $p$ in $\partial D_{1}$ and $q$ in $\partial D_{2}$ such that $F$ extends to a biholomorphism in a neighborhood of $p$ with $F(p)=q$.

(iv) $F$ is algebraic.

The tools used to prove Proposition 1.2, developed in different papers and valid for decoupled domains, are the existence of peak plurisubharmonic functions with algebraic growth (constructed in [13]) implying an equivalence distance property for $f$ (part (i)), and the existence of peak holomorphic functions at infinity for rigid domains, given by [2] (part (iii)). Part (ii) uses the transversality of the Segre varieties, satisfied in any dimension and part (iv) is an immediate consequence of part (iii) by $[\mathbf{1 9}]$. The complete proof of Proposition 1.2 is given by Proposition 1.5 and Lemma 2.2 of [8].

Without changing the properties of $D_{2}$, we may assume that $q=0$.

The map $F$ inherits some properties from $f$, since the family $\left(F^{\nu}\right)_{\nu}$ satisfies the following uniform properties: 
Proposition 1.3. Let a be a point in $\partial D_{1}$ and $\left(a^{\nu}\right)_{\nu}$ a sequence of points in $\partial \Omega_{1}^{\nu}$, converging to a.

(i) If $\left(F^{\nu}\left(a^{\nu}\right)\right)_{\nu}$ is bounded then there exists a neighborhood $U$ of a in $\mathbb{C}^{n+1}$ and a real positive constant $C$ such that for every positive integer $\nu$ and every $z, z^{\prime}$ in $\Omega_{1}^{\nu} \cap U$ we get:

$$
\left|F^{\nu}(z)-F^{\nu}\left(z^{\prime}\right)\right| \leq C\left|z-z^{\prime}\right|^{(1 / 2 k)} .
$$

(ii) If $\lim _{\nu \rightarrow \infty}\left|F^{\nu}\left(a^{\nu}\right)\right|=+\infty$ then $\lim _{\substack{z \in \bar{D}_{1} \\ z \rightarrow a}}|F(z)|=+\infty$.

Proof: See Propositions 4.1 and 5.1 of [10].

We deduce from Propositions 1.2 and 1.3 the following result:

Proposition 1.4. If $f$ satisfies the assumptions of Theorem 1 then the set $f^{-1}(f(0))$ is compact near the origin in $\Gamma_{1}$ and $f$ is a $\mathcal{C}^{\infty}$ locally finite map at the origin under one of the following conditions:

(i) $F(0)$ is a finite point in $\partial D_{2}$.

(ii) $\lim _{\substack{z \in \bar{D}_{1} \\ z \rightarrow 0}}|F(z)|=+\infty$ and $F^{-1}(\infty)$ is a finite set in $\partial D_{1}$.

Proof of Proposition 1.4: Assume by contradiction that the set $f^{-1}(f(0))$ is not compact near the origin. Then there is, for every sufficiently small real positive number $r$, a point in $f^{-1}(f(0))$ of modulus $r$. Using the expression of the dilation we can find for every $\nu, k$ larger than or equal to one a point $a_{k}^{\nu}$ in $\partial \Omega_{1}^{\nu}$ satisfying: $F^{\nu}\left(a_{k}^{\nu}\right)=F^{\nu}(0),\left|a_{k}^{\nu}\right|=\frac{1}{k}$. We may assume that for every $k$ the sequence $\left(a_{k}^{\nu}\right)_{\nu}$ converges to a point $a_{k}$ in $\partial D_{1}$ with modulus $1 / k$ : the sequence $\left(a_{k}\right)_{k}$ converges to 0 in $\partial D_{1}$.

Part (i): by the assumption and Proposition 1.3 part (ii) the sequence $\left(F^{\nu}(0)\right)_{\nu}$ is bounded. Then by Proposition 1.3 part (i) and Proposition 1.1 part (iii) this sequence converges to $F(0)$. The condition $F\left(a_{k}\right)=F(0)$ contradicts Proposition 1.2 part (ii).

Part (ii): the non compactness of $f^{-1}(f(0))$ implies that $\lim _{\nu \rightarrow \infty}\left|F^{\nu}\left(a_{k}^{\nu}\right)\right|=+\infty$ for every $k$ and thus by Proposition 1.3 part (ii) that the point $a_{k}$ belongs to $F^{-1}(\infty)$ for every $k$ : this contradicts the finiteness of $F^{-1}(\infty)$. The smoothness of $f$ is then given by [4].

According to Proposition 1.4 one needs to answer the two following questions to prove Theorem 1:

1. When is $F(0)$ a finite point in $\partial D_{2}$ ?

2. What is the structure of the set $F^{-1}(\infty)$ if $\lim _{\substack{z \in D_{1} \\ z \rightarrow 0}}|F(z)|=+\infty$ ? 
In the next section we describe the CR infinitesimal automorphisms of $\partial D_{1}$. We use this description in Section 3 to answer question 1. The answer to question 2 is given in Section 4 .

\section{Classification of vector fields}

Since we deal with decoupled real hypersurfaces we start this section with some results on real hypersurfaces in complex dimension two. Let $H$ be a real subharmonic non harmonic homogeneous polynomial defined in $\mathbb{C}$, and $\Gamma$ be the real hypersurface defined by: $\Gamma=\left\{\left(z_{0}, z_{1}\right) \in \mathbb{C}^{2}\right.$ : $\left.\operatorname{Im} z_{0}+H\left(z_{1}, \overline{z_{1}}\right)=0\right\}$. The real dimension of the real vector space of CR infinitesimal automorphisms at a strictly pseudoconvex point of $\Gamma$ is equal to 2, 3 or 8 according to a result of E. Cartan [6]. This equals 8 if $\Gamma$ is spherical, in which case we may assume that $H\left(z_{1}, \overline{z_{1}}\right)=\left|z_{1}\right|^{2 m}$, and 3 if $\Gamma$ is a tube hypersurface (and $\left.H\left(z_{1}, \overline{z_{1}}\right)=\left(\operatorname{Im} z_{1}\right)^{2 m}\right)$.

We assume in this section that the hypersurface $\partial D_{1}$ has at least one non spherical direction at point $p$ given by Proposition 1.2. This means that if we write $p=\left(p_{0}, \ldots, p_{n}\right)$ then there is an integer $l$ satisfying $0 \leq l \leq n-1$ such that for every integer $j$ larger than $l$ the hypersurface $\Gamma_{j}=\left\{\left(z_{0}, z_{j}\right) \in \mathbb{C}^{2}: \operatorname{Im} z_{0}+H_{j}\left(z_{j}, \overline{z_{j}}\right)=-\sum_{k \neq j} H_{k}\left(p_{k}, \overline{p_{k}}\right\}\right.$ is not biholomorphic to the unit sphere at $\left(p_{0}, p_{j}\right)$. In the following $l$ denotes the smallest integer satisfying this condition and we remark that the equality $l=0$ means that $\partial D_{1}$ is a spherical hypersurface in $\mathbb{C}^{n+1}$. The infinitesimal $\mathrm{CR}$ automorphisms of $\partial D_{1}$ are then given by the following proposition:

Proposition 2.1. Let $p$ be given by Proposition 1.2 and let $X=$ $\sum_{j=0}^{n} X_{j} \frac{\partial}{\partial z_{j}}$ be a CR infinitesimal automorphism at $p$.

- Case 1: $l=0$.

There exist $(n+2)$ real constants $a_{0}, b_{0}, b_{1}, \ldots, b_{n}$ such that:

$$
\left\{\begin{array}{l}
X_{0}\left(z_{0}, z\right)=a_{0}+b_{0} z_{0}+2 \sum_{k=1}^{n} m_{k} b_{k} z_{k}^{2 m_{k}-1} \\
X_{j}\left(z_{0}, z\right)=2 m_{j} b_{0} z_{j}-\frac{i}{2} b_{j}
\end{array} \quad \forall j \geq 1 .\right.
$$

- Case 2: $l>0$.

There exist $(n+2)$ real constants $a_{0}, b_{0}, b_{1}, \ldots, b_{n}$ and $l(l-1)$ complex constants $c_{j k}$ defined for $1 \leq j, k \leq l$ with $j \neq k$ such that:

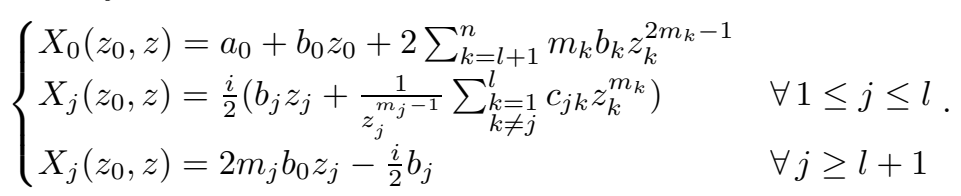


Proof of Proposition 2.1: Since case 1 may be considered as a special case of case 2 , let us study case 2 . We may assume that for every integer $k$ between 1 and $l$ the polynomial $H_{k}$ is defined for $z_{k}$ in $\mathbb{C}$ by: $H_{k}\left(z_{k}, \overline{z_{k}}\right)=\left|z_{k}\right|^{2 m_{k}}$. Let $j$ be an integer between $l+1$ and $n$. Fixing all the variables $z_{k}=p_{k}$ for $k$ different from $j(1 \leq k \leq n)$ the vector field

$$
X^{j}\left(z_{0}, z_{j}\right)=\left(X_{0}\left({ }^{j} z\right)-2 i \sum_{\substack{k \neq j \\ k=1}}^{n} \frac{\partial H_{k}}{\partial z_{k}}\left(p_{k}, \overline{p_{k}}\right) X_{k}\left({ }^{j} z\right)\right) \frac{\partial}{\partial z_{0}}+X_{j}\left({ }^{j} z\right) \frac{\partial}{\partial z_{j}},
$$

where ${ }^{j} z=\left(z_{0}, p_{1}, \ldots, p_{j-1}, z_{j}, p_{j+1}, \ldots, p_{n}\right)$, is tangent to the hypersurface

$$
S^{j}=\left\{\left(z_{0}, z_{j}\right) \in \mathbb{C}^{2}: \operatorname{Im} z_{0}+H_{j}\left(z_{j}, \overline{z_{j}}\right)=-\sum_{k \neq j} H_{k}\left(p_{k}, \overline{p_{k}}\right)\right\}
$$

at $\left(p_{0}, p_{j}\right)$. As $S^{j}$ is not spherical, every local tangent vector field extends to a global one acording to $[6]$. Then, because of the homogeneity of $S^{j}$, each homogeneous part in the expansion of $X^{j}$ is also a CR infinitesimal automorphism for $S^{j}$. Since $S^{j}$ is of finite type the real vector space of such vector fields has a finite dimension; thus $X^{j}$ is a polynomial vector field which means that all the coefficients of $X^{j}$ are polynomial. Hence the vector field $\tilde{X}$ defined for $\left(z_{0}, z_{l+1}, \ldots, z_{n}\right)$ in a neighborhood of $\left(p_{0}, p_{l+1}, \ldots, p_{n}\right)$ in $\mathbb{C}^{n-l+1}$ by:

$$
\begin{array}{r}
\tilde{X}\left(z_{0}, z_{l+1}, \ldots, z_{n}\right)=\left(X_{0}\left(z^{l}\right)-2 i \sum_{k=1}^{l} \frac{\partial H_{k}}{\partial z_{k}}\left(p_{k}, \overline{p_{k}}\right) X_{k}\left(z^{l}\right)\right) \frac{\partial}{\partial z_{0}} \\
+\sum_{k=l+1}^{n} X_{k}\left(z^{l}\right) \frac{\partial}{\partial z_{k}}
\end{array}
$$

where $z^{l}=\left(z_{0}, p_{1}, \ldots, p_{l}, z_{l+1}, \ldots, z_{n}\right)$, is polynomial.

Let us expand $\tilde{X}$ in homogeneous vector fields with respect to the weights $2 m_{l+1}, \ldots, 2 m_{n}$ (we recall that $2 m_{j}$ is the degree of polynomial $H_{j}$ given by Proposition 1.1) with the convention that $\partial / \partial z_{j}$ has weight $-1 / 2 m_{j}$. The classification of homogeneous vector fields $Q$ tangent to $\tilde{S}=\left\{\left(z_{0}, z_{l+1}, \ldots, z_{n}\right) \in \mathbb{C}^{n-l+1}: \operatorname{Im} z_{0}+\sum_{j=l+1}^{n} H_{j}\left(z_{j}, \overline{z_{j}}\right)=\right.$ $\left.-\sum_{j=1}^{l}\left|p_{j}\right|^{2 m_{j}}\right\}$ is given by [3] (Lemmas 2.7, 3.4, 3.5). Since there is no spherical direction in $\tilde{S}$ the admissible weights for these vector fields are -1 (corresponding to the translation $\left.\frac{\partial}{\partial z_{0}}\right),-1 / 2 m_{j}($ with $l+1 \leq j \leq n)$ 
and 0 . Moreover the only $\mathrm{CR}$ infinitesimal automorphism of weight 0 corresponds to the dilation:

$$
Q\left(z_{0}, z\right)=z_{0} \frac{\partial}{\partial z_{0}}+\sum_{k=1}^{n} \frac{1}{2 m_{k}} z_{k} \frac{\partial}{\partial z_{k}} .
$$

A straightforward computation based on Lemma 2.7 of [3] gives the form of the homogeneous CR infinitesimal automorphisms of weight $-1 / 2 m_{j}$ and consequently of the CR infinitesimal automorphisms of weight $w t(Q)$ with $-1<w t(Q)<0$ :

$$
Q\left(z_{0}, z\right)=\sum_{k=1}^{n} b_{k}\left(2 m_{k} z_{k}^{2 m_{k}-1} \frac{\partial}{\partial z_{0}}-\frac{i}{2} \frac{\partial}{\partial z_{k}}\right), \quad b_{k} \in \mathbb{R} .
$$

Thus we have $\tilde{X}=\tilde{X}_{0} \frac{\partial}{\partial z_{0}}+\sum_{j=l+1}^{n} \tilde{X}_{j} \frac{\partial}{\partial z_{j}}$ with:

$$
\left\{\begin{array}{l}
\tilde{X}_{0}\left(z_{0}, z_{l+1}, \ldots, z_{n}\right)=a_{0}+b_{0} z_{0}+2 \sum_{k=l+1}^{n} m_{k} b_{k} z_{k}^{2 m_{k}-1} \\
\tilde{X}_{j}\left(z_{0}, z_{l+1}, \ldots, z_{n}\right)=2 m_{j} b_{0} z_{j}-\frac{i}{2} b_{j}, \quad \forall l+1 \leq j \leq n
\end{array}\right.
$$

where $a_{0}, b_{0}, b_{l+1}, \ldots, b_{n}$ are real analytic real functions of the variables $z_{1}, \ldots, z_{l}$, defined locally at $\left(p_{1}, \ldots, p_{l}\right)$. Thus the vector field $X$ satisfies the following system:

$$
\left\{\begin{aligned}
X_{0}\left(z_{0}, z\right) & -2 i \sum_{k=1}^{l}\left(m_{k} \bar{z}_{k}^{m_{k}} z_{k}^{m_{k}-1}\right) X_{k}\left(z_{0}, z\right) & & \\
& =a_{0}+b_{0} z_{0}+2 \sum_{k=l+1}^{n} m_{k} b_{k} z_{k}^{2 m_{k}-1} & & \\
X_{j}\left(z_{0}, z\right) & =2 m_{j} b_{0} z_{j}-\frac{i}{2} b_{j}, & & \forall l+1 \leq j \leq n
\end{aligned}\right.
$$

for $\left(z_{0}, z\right)$ close to $p$. Since the vector field $X$ is holomorphic it follows from the second equation of system $(\mathrm{S})$ that $b_{0}, b_{l+1}, \ldots, b_{n}$ are real constants.

Let us fix an integer $j$ such that $1 \leq j \leq l$.

Differentiating the first equation of system $(\mathrm{S})$ with respect to $\overline{z_{j}}$ we have:

$$
-2 i m_{j}^{2} \bar{z}_{j}^{m_{j}-1} z_{j}^{m_{j}-1} X_{j}=\bar{\partial}_{j} a_{0}
$$

Since $X_{j}$ is holomorphic there exist:

- A holomorphic function $f_{j}$ of the variables $z_{1}, \ldots, z_{l}$ such that $f_{j \mid\left\{z_{j}=0\right\}}=0$.

- A holomorphic function $\tilde{f}_{j}$ depending on the variables $z_{k}$ for $1 \leq$ $k \leq l, k \neq j$. 
- A real analytic function $g_{j}$ not depending on $\bar{z}_{j}$ such that $a_{0}=$ $\bar{z}_{j} m_{j}\left(f_{j}+\tilde{f}_{j}\right)+g_{j}$.

Since $a_{0}$ is a real function and $f_{j}$ is holomorphic we have $f_{j}\left(z_{1}, \ldots, z_{l}\right)=$ $\alpha_{j} z_{j}^{m_{j}}$ where $\alpha_{j}$ is a real constant and $g_{j}=z_{j}^{m_{j}} \tilde{f}_{j}+\tilde{g}_{j}$ where $\tilde{g}_{j}$ is a real analytic real function depending neither on $z_{j}$ nor on $\overline{z_{j}}$. Then $a_{0}=\alpha_{j}\left|z_{j}\right|^{2 m_{j}}+\left(\overline{z_{j}} m_{j} \tilde{f}_{j}+z_{j}^{m_{j}} \overline{\tilde{f}_{j}}\right)+\tilde{g_{j}}$. Replacing $X_{1}, \ldots, X_{l}$ in terms of $\bar{\partial}_{j} a_{0}$ (see equation (1)) in the first equation of system (S) we obtain the following equality:

$$
\begin{aligned}
X_{0}\left(z_{0}, z\right)=-\sum_{\substack{k=1 \\
k \neq j}}^{l}\left(\alpha_{k}\left|z_{k}\right|^{2 m_{k}}+\bar{z}_{k}^{m_{k}} \tilde{f}_{k}\right)+z_{j}^{m_{j}} & \overline{\tilde{f}}_{j}+\tilde{g}_{j}+b_{0} z_{0} \\
& +2 \sum_{k=l+1}^{n} m_{k} b_{k} z_{k}^{2 m_{k}-1}
\end{aligned}
$$

and adding these $l$ equalities for $1 \leq j \leq l$ :

$$
\begin{array}{r}
l X_{0}=-(l-1) \sum_{k=1}^{l}\left(\alpha_{k}\left|z_{k}\right|^{2 m_{k}}\right)-l \sum_{k=1}^{l} \bar{z}_{k}^{m_{k}} \tilde{f}_{k}+\sum_{k=1}^{l}\left(\bar{z}_{k}^{m_{k}} \tilde{f}_{k}+z_{k}^{m_{k}} \overline{\tilde{f}}_{k}\right) \\
+\sum_{k=1}^{l} \tilde{g_{k}}+l b_{0} z_{0}+2 l \sum_{k=l+1}^{n} m_{k} b_{k} z_{k}^{2 m_{k}-1} .
\end{array}
$$

Let us identify the holomorphic terms in this expression. Since $\sum_{k=1}^{l} \bar{z}_{k}^{m_{k}} \tilde{f}_{k}$ is not holomorphic, this is a real function and we have:

$$
\left\{\begin{array}{c}
l X_{0}=a+l b z_{0}+2 l \sum_{k=l+1}^{n} m_{k} b_{k} z_{k}^{2 m_{k}-1} \\
\sum_{k=1}^{l} \tilde{g}_{k}=(l-1) \sum_{k=1}^{l}\left(\alpha_{k}\left|z_{k}\right|^{2 m_{k}}\right)+l \sum_{k=1}^{l} \bar{z}_{k}^{m_{k}} \tilde{f}_{k} \\
\quad+\sum_{k=1}^{l}\left(\bar{z}_{k}^{m_{k}} \tilde{f}_{k}+z_{k}^{m_{k}} \tilde{\tilde{f}}_{k}\right)
\end{array}\right.
$$

Consequently $\sum_{k=1}^{l} \bar{z}_{k}^{m_{k}} \tilde{f}_{k}=\sum_{k=1}^{l} z_{k}^{m_{k}} \tilde{\tilde{f}_{k}}$ and for every $1 \leq j \leq l$ :

$$
\tilde{f}_{j}=\sum_{k \neq j} z_{k}^{m_{k}} \overline{\left(\frac{\partial_{j} \tilde{f}_{k}}{m_{j} z_{j}^{m_{j}-1}}\right)} .
$$


Since $\tilde{f}_{j}$ is holomorphic in $z_{k}$ for $1 \leq k \leq l, k \neq j$ it follows that $z_{k}^{m_{k}} \overline{\left(\frac{\partial_{j} \tilde{f}_{k}}{m_{j} z_{j}^{m_{j}-1}}\right)}$ is holomorphic: there is a complex constant $a_{j k}$ such that $\partial_{j} \tilde{f}_{k}=\overline{a_{j k}} m_{j} z_{j}^{m_{j}-1}$ or:

$$
\tilde{f}_{j}=\sum_{k \neq j} c_{j k} z_{k}^{m_{k}} .
$$

It is then sufficient to set $b_{j}=\alpha_{j} / m_{j}$ and $c_{j k}=a_{j k} / m_{j}$ for $1 \leq j \leq l$, $1 \leq k \leq l, k \neq j$.

\section{Classification of maps from $D_{1}$ to $D_{2}$}

We recall that the limit map $F$ obtained in Section 1 is locally proper according to Proposition 1.2 part (i). We assume in this section that $\partial D_{1}$ has at least one non spherical direction and we use the description of $\mathrm{CR}$ infinitesimal automorphisms given by Proposition 2.1 to obtain the following:

Proposition 3.1. $F(0)$ is a finite point in $\partial D_{2}$.

Proof of Proposition 3.1: According to Proposition 1.2 parts (iii)-(iv) the map $G=F^{-1}$, locally defined at 0 with $G(0)=p$, is an algebraic map. Since the vector field $G_{\star}\left(\frac{\partial}{\partial z_{0}}\right)$ is a CR infinitesimal automorphism at $p$ the map $G$ satisfies:

- In case 1 of Proposition 2.1:

$$
\begin{cases}\frac{\partial G_{0}}{\partial z_{0}}=a_{0}+b_{0} G_{0}+2 \sum_{k=1}^{n} m_{k} b_{k} G_{k}^{2 m_{k}-1} & \\ \frac{\partial G_{j}}{\partial z_{0}}=2 m_{j} b_{j} G_{j}-\frac{i}{2} b_{j} & \forall j \in\{1, \ldots, n\}\end{cases}
$$

- In case 2 of Proposition 2.1:

(S2) $\left\{\begin{array}{lll}\frac{\partial G_{0}}{\partial z_{0}}=a_{0}+b_{0} G_{0}+2 \sum_{k=l+1}^{n} m_{k} b_{k} G_{k}^{2 m_{k}-1} & & \\ \frac{\partial G_{j}}{\partial z_{0}}=\frac{i}{2}\left(b_{j} G_{j}+\sum_{\substack{k=1 \\ k \neq j}}^{l} c_{j k} \frac{G_{k}^{m_{k}}}{G_{j}^{m_{j}-1}}\right) & & \forall j \in\{1, \ldots, l\} \\ \frac{\partial G_{j}}{\partial z_{0}}=2 m_{j} b_{0} G_{j}-\frac{i}{2} b_{j} & & \forall j \in\{l+1, \ldots, n\}\end{array}\right.$. 
Since system (S1) can be considered as a subsystem of system (S2) we will focus on the resolution of system (S2). The map $G$ being algebraic the resolution of the equation $\frac{\partial G_{j}}{\partial z_{0}}=2 m_{j} b_{0} G_{j}-\frac{i}{2} b_{j}$ implies that $b_{0}=0$. Moreover there exist functions $\tilde{G}_{0}, \ldots, \tilde{G}_{n}, \tilde{G}_{j k}(1 \leq j \leq l, 0 \leq k \leq l-1)$, holomorphic in a neighborhood $U^{\prime}$ of 0 in $\mathbb{C}^{n}$, such that:

(S3) $\left\{\begin{array}{ll}G_{0}\left(z_{0}, z\right)=a z_{0} & \\ \quad+2 i \sum_{l+1}^{n}\left(\frac{-i}{2} b_{j} z_{0}+\tilde{G}_{j}(z)\right)^{2 m_{j}}+\tilde{G}_{0}(z) & \\ \left(G_{j}\left(z_{0}, z\right)\right)^{m_{j}}=\sum_{k=0}^{l-1} \tilde{G}_{j k}(z) z_{0}^{k} & \forall j \in\{1, \ldots, l\} \\ G_{j}\left(z_{0}, z\right)=\frac{-i}{2} b_{j} w+\tilde{G}_{j}(z) & \forall j \in\{l+1, \ldots, n\}\end{array}\right.$.

Let us write for every integer $j$ in $\{1, \ldots, n\} H_{j}\left(z_{j}+p_{j}, \bar{z}_{j}+\bar{p}_{j}\right)=$ $Q_{j}\left(z_{j}, \bar{z}_{j}\right)+\operatorname{Im}\left(S_{j}\left(z_{j}, \bar{z}_{j}\right)\right)+H_{j}\left(p_{j}, \bar{p}_{j}\right)$ where $Q_{j}$ is a real subharmonic polynomial without harmonic term and $S_{j}$ is a holomorphic polynomial without constant term. If $T$ is the transformation:

$$
T\left(z_{0}, z\right)=\left(z_{0}-p_{0}+\sum_{j=1}^{n} S_{j}\left(z_{j}-p_{j}, \bar{z}_{j}-\bar{p}_{j}\right), z_{1}-p_{1}, \ldots, z_{n}-p_{n}\right)
$$

and $D_{1}^{\prime}$ is the domain $D_{1}^{\prime}=\left\{\left(z_{0}, z\right) \in \mathbb{C}^{n+1}: \operatorname{Im} z_{0}+\sum_{j=1}^{n} Q_{j}\left(z_{j}, \bar{z}_{j}\right)<\right.$ $0\}$ then we have the equivalence:

$$
\left(z_{0}, z\right) \in D_{1} \Leftrightarrow T\left(z_{0}, z\right) \in D_{1}^{\prime} .
$$

Let us denote $T \circ G=g=\left(g_{0}, \ldots, g_{n}\right)$. By assumption $g(0)=0$. For every $z^{0}$ in $U^{\prime}$ the function $g$ is well defined on the half plane $\Lambda_{z^{0}}=$ $\left\{\left(z_{0}, z\right) \in \mathbb{C}^{n+1}: \operatorname{Im} z_{0}<-\sum_{j=1}^{n} P_{j}\left(z_{j}^{0}, \overline{z_{j}^{0}}\right), z=z^{0}\right\}$ and $\left(F \circ T^{-1}\right) \circ g$ is the identity in a neighborhood of the origin on $\Lambda_{z^{0}}$.

Assume that there is a point $\left(z_{0}^{0}, z^{0}\right)$ on $\Lambda_{z^{0}}$ and a path $\gamma$ in $\Lambda_{z^{0}}$ connecting 0 and $\left(z_{0}^{0}, z^{0}\right)$ (i.e. $\gamma(0)=0, \gamma(1)=\left(z_{0}^{0}, z^{0}\right)$ ) such that $g(\gamma(t))$ belongs to $D_{1}^{\prime}$ for $t$ in $] 0,1\left[\right.$ and $\lim _{t \rightarrow 1} g(\gamma(t)) \in \partial D_{1}^{\prime}$. Since $\left(F \circ T^{-1}\right) \circ g=$ id in a neighborhood of $\gamma(] 0,1[)$ we may assume that $g(\gamma(t))$ converges to a finite point in $\partial D_{1}^{\prime}$ when $t$ converges to 1 . Its image by the local proper map $F \circ T^{-1}$ (see Proposition $\left.1.2(\mathrm{i})\right)$ is $\left(z_{0}^{0}, z^{0}\right)$ : this is a contradiction and thus the set $g\left(\Lambda_{z^{0}}\right)$ is contained in $D_{1}^{\prime}$.

Assume now that either $\tilde{G}_{j k}\left(z^{0}\right) \neq 0$ for some $(j, k)$ with $1 \leq j \leq l$, $0 \leq k \leq l-1$ or $b_{j} \neq 0$ for some $j$ with $l+1 \leq j \leq n$. According to system (S3) the restriction of $g$ to $\Lambda_{z^{0}}$ is a polynomial map of the variable $z_{0}$ and the restriction of the function $\operatorname{Im} g_{0}+\sum_{j=1}^{n} Q_{j}\left(g_{j}, \bar{g}_{j}\right)$ to $\Lambda_{z^{0}}$ is a negative subharmonic polynomial function. Its weighted 
homogeneous part of largest degree, given by the terms of largest degree of the polynomial

$$
\begin{aligned}
2 \operatorname{Im} \sum_{j=l+1}^{n}\left(\frac{-i}{2} b_{j} z_{0}\right)^{2 m_{j}}+\sum_{j=1}^{l}\left|\sum_{k=0}^{l-1} \tilde{G}_{j k}\left(z^{0}\right) z_{0}^{k}\right|^{2 m_{j}} & \\
& +\sum_{j=l+1}^{n} H_{j}\left(\frac{-i}{2} b_{j} z_{0}, \frac{i}{2} b_{j} \overline{z_{0}}\right)
\end{aligned}
$$

is a subharmonic non harmonic function on $\Lambda_{z^{0}}$. By Lemma 1.2 of [2] this is positive in some directions. Since its limit at infinity in these directions is infinite it follows that $\operatorname{Im} g_{0}+\sum Q_{j}\left(g_{j}, \bar{g}_{j}\right)$ is not a negative function on $\Lambda_{z^{0}}$ : this is a contradiction.

Consequently $b_{j}=0$ for $l+1 \leq j \leq n$ and $\tilde{G}_{j k}$ is identically zero on $U^{\prime}$ for $1 \leq j \leq l, 0 \leq k \leq l-1$. Then $g\left(z_{0}, z\right)=\left(a z_{0}+\right.$ $\left.h_{0}(z), h_{1}(z), \ldots, h_{n}(z)\right)$, where $h_{0}, h_{1}, \ldots, h_{n}$ are holomorphic functions defined locally at 0 in $\mathbb{C}^{n}$. Moreover there is a real analytic function $\lambda$ defined in a neighborhood of 0 in $\mathbb{C}^{n+1}$ with $\lambda(0)=0$ such that we get locally at 0 :

$\operatorname{Im} g_{0}\left(z_{0}, z\right)+\sum_{j=1}^{n} Q_{j}\left(g_{j}\left(z_{j}\right), \bar{g}_{j}\left(z_{j}\right)\right)=\lambda\left(z_{0}, z\right)\left(\operatorname{Im} z_{0}+\sum_{j=1}^{n} P_{j}\left(z_{j}, \bar{z}_{j}\right)\right)$.

By setting $z_{0}=0$ in this equation we obtain, since $h_{j}(0)=0$ and $Q_{j}$, $P_{j}$ have no harmonic terms, that $h_{0}$ is identically zero. Thus we get: $g\left(z_{0}, z\right)=\left(a z_{0}, h_{1}(z), \ldots, h_{n}(z)\right)$, with $h_{j}(0)=0$. According to the implicit function theorem, there is a complex constant $a^{\prime}$ and holomorphic functions $\tilde{f}_{1}, \ldots, \tilde{f}_{n}$, locally defined at the origin, such that:

$$
\left(F \circ T^{-1}\right)\left(z_{0}, z\right)=\left(a^{\prime} z_{0}, \tilde{f}_{1}(z), \ldots, \tilde{f}_{n}(z)\right) \text {. }
$$

Since $F \circ T^{-1}$ is globally defined on $D_{1}^{\prime}$, the functions $\tilde{f}_{1}, \ldots, \tilde{f}_{n}$ are holomorphic on $\mathbb{C}^{n}$. For every $z=\left(z_{1}, \ldots, z_{n}\right)$ in $\mathbb{C}^{n}$, the point $\left(-i \sum_{j=1}^{n} Q_{j}\left(z_{j}, \overline{z_{j}}\right), z\right)$ belongs to $\partial D_{1}^{\prime}$ and so the point $F \circ T^{-1}\left(\left(-i \sum_{j=1}^{n} Q_{j}\left(z_{j}, \overline{z_{j}}\right), z\right)\right)$ belongs to $\partial D_{2}$. Thus we have for $z=$ $\left(z_{1}, \ldots, z_{n}\right)$ in $\mathbb{C}^{n}$ :

$$
\sum_{j=1}^{n} P_{j}\left(\tilde{f}_{j}(z), \overline{\tilde{f}_{j}(z)}\right)=\operatorname{Im}\left(a^{\prime}\right) \sum_{j=1}^{n} Q_{j}\left(z_{j}, \overline{z_{j}}\right) .
$$

Let $k$ be an integer between 1 and $n$ and, for every $l$ different from $k, z_{l}^{0}$ be a fixed complex number. If we set $z^{k}=\left(z_{1}^{0}, \ldots, z_{k-1}^{0}, z_{k}, z_{k+1}^{0}, \ldots, z_{n}^{0}\right)$ then, for every integer $j$ in $\{1, \ldots, n\}$, the function $z_{k} \mapsto P_{j}\left(\tilde{f}_{j}\left(z^{k}\right), \overline{\tilde{f}_{j}\left(z^{k}\right)}\right)$ 
is subharmonic on $\mathbb{C}$ and its Laplacian has an algebraic growth; this is a subharmonic polynomial. So there are an integer $k_{0}$ and complex constants $\alpha_{s, t}$, defined for $(s, t)$ with $1 \leq s, t \leq k_{0}$, such that:

$$
P_{j}\left(\tilde{f}_{j}(z), \overline{\tilde{f}_{j}(z)}\right)=\sum_{1 \leq s, t \leq k_{0}} \alpha_{s, t} z^{s} \bar{z}^{t} .
$$

If $P_{j}\left(z_{j}, \overline{z_{j}}\right)=\sum_{1 \leq s, t \leq 2 k} a_{s, t} z_{j}^{s}{\overline{z_{j}}}^{t}$ a polarization of equation (2) implies for $\left(z_{k}, \zeta\right)$ in $\mathbb{C}^{2}$ :

$$
\sum_{1 \leq s, t \leq 2 k} a_{s, t}\left(\tilde{f}_{j}\left(z^{k}\right)\right)^{s}\left(\tilde{\tilde{f}}_{j}\left(\bar{\zeta}^{k}\right)\right)^{t}=\sum_{1 \leq s, t \leq k_{0}} \alpha_{s, t} z_{k}^{s} \zeta^{t}
$$

with $\zeta^{k}=\left(z_{1}^{0}, \ldots, z_{k-1}^{0}, \zeta, z_{k+1}^{0}, \ldots, z_{n}^{0}\right)$.

Hence $\sum_{1 \leq s, t \leq 2 k} a_{s, t} \frac{d^{k_{0}+1}}{d z^{k_{0}+1}}\left(\left(\tilde{f}_{j}\left(z^{k}\right)\right)^{s}\right)\left(\tilde{\tilde{f}}_{j}\left(\bar{\zeta}^{k}\right)\right)^{t}$ is identically zero on $\mathbb{C}^{2}$. Considering this as a polynomial in $\tilde{f}_{j}\left(\bar{\zeta}^{k}\right)$ and since $P_{j}$ is not identically zero, there is an integer $s$ between 1 and $2 k$ such that $\frac{d^{k_{0}+1}}{d z^{k_{0}+1}}\left(\left(\tilde{f}_{j}\left(z^{k}\right)\right)^{s}\right)$ is identically zero on $\mathbb{C}$ : the map $z_{k} \mapsto\left(\tilde{f}_{j}\left(z^{k}\right)\right)^{s}$ is a polynomial. Hence the holomorphic function $z_{k} \mapsto \tilde{f}_{j}\left(z^{k}\right)$, globally defined on $\mathbb{C}$, is polynomial and thus the map $F \circ T$ is polynomial. Finally the point $F(0)=$ $(F \circ T)\left(T^{-1}(0)\right)$ is a finite point in $\partial D_{2}$. This proves Proposition 3.1.

\section{The end of the proof of Theorem 1}

We proved in Section 3 that if $\partial D_{1}$ has at least one non spherical direction then $F(0)$ is a finite point. Theorem 1 is then a consequence of Proposition 1.4. Assume now that the hypersurface $\partial D_{1}$ is spherical at point $p$. We may write: $D_{1}=\left\{\left(z_{0}, z\right) \in \mathbb{C}^{n+1}: \operatorname{Im} z_{0}+\sum_{j=1}^{n}\left|z_{j}\right|^{2 m_{j}}<\right.$ $0\}$. By Proposition 1.2 part (iii) the domain $D_{2}$ is spherical at the origin. Hence by a work of A. V. Isaev [15] the polynomial $P=\sum_{j=1}^{n} P_{j}$ satisfies a system of partial differential equations:

$$
\frac{\partial^{2} P}{\partial z_{j} \partial z_{k}}=\sum_{l=1}^{n} \frac{\partial P}{\partial z_{l}}\left(E^{l} \frac{\partial P}{\partial z_{j}} \frac{\partial P}{\partial z_{k}}+D_{j}^{l} \frac{\partial P}{\partial z_{k}}+D_{k}^{l} \frac{\partial P}{\partial z_{j}}+C_{j k}^{l}\right)+H_{j k}
$$

where $E^{l}, D_{j}^{l}, C_{j k}^{l}$ and $H_{j k}$ are holomorphic functions defined in a neighborhood of the origin. It follows by setting $z_{k}=0$ for $k \neq j$ that the polynomial $P_{j}$ satisfies an equivalent system and the domain $\left\{\left(z_{0}, z_{j}\right) \in\right.$ $\left.\mathbb{C}^{2}: \operatorname{Im} z_{0}+P_{j}\left(z_{j}, \bar{z}_{j}\right)<0\right\}$ is also spherical in $\mathbb{C}^{2}$ for every $j$ in $\{1, \ldots, n\}$ by Proposition 2.2 of [15]. Since $P_{j}$ has no pure anti-holomorphic term 
we obtain by a comparison of the terms of maximal degree in $\overline{z_{j}}$ that $E^{j}=D_{j}^{j}=H_{j j}=0$. Thus $P_{j}$ satisfies the following system in $\mathbb{C}^{2}$ :

$$
\frac{\partial^{2} P_{j}}{\partial z_{j}^{2}}\left(z_{j}, \overline{z_{j}}\right)=C_{j j}^{j}\left(z_{j}\right) \frac{\partial P_{j}}{\partial z_{j}}\left(z_{j}, \overline{z_{j}}\right)
$$

where $C_{j j}^{j}$ is a holomorphic function. An integration shows that there exists a holomorphic polynomial $h_{j}$ such that $P_{j}\left(z_{j}, \overline{z_{j}}\right)=\left|h_{j}\left(z_{j}\right)\right|^{2}$. Since $D_{1}$ is biholomorphic to the ellipsoïd $\left\{\left(z_{0}, z\right) \in \mathbb{C}^{n+1}:\left|z_{0}\right|^{2}+\right.$ $\left.\sum_{j=1}^{n}\left|z_{j}\right|^{2 m_{j}}<1\right\}$ one can prove as in Proposition 3.1 of [8] that the map $F$ is a proper map from $D_{1}$ to $D_{2}$. Thus if $g^{1}$ and $g^{2}$ are the maps defined in $\mathbb{C}^{n+1}$ by $g^{1}:\left(z_{0}, z\right) \mapsto\left(z_{0}, z_{1}^{m_{1}}, \ldots, z_{n}^{m_{n}}\right)$ and $g^{2}:\left(z_{0}, z\right) \mapsto$ $\left(z_{0}, h_{1}\left(z_{1}\right), \ldots, h_{n}\left(z_{n}\right)\right)$, there is a proper holomorphic auto correspondence $\tilde{F}$ of the unbounded representation $\mathbb{H}$ of the unit ball of $\mathbb{C}^{n+1}$ such that: $\tilde{F} \circ g^{1}=g^{2} \circ F([\mathbf{1}])$. Every component of its graph is the graph of an automorphism of $\mathbb{H}$. Since $F^{-1}(\infty)$ is contained in $\left(\tilde{F} \circ g^{1}\right)^{-1}(\infty)$ and every automorphism of $\mathbb{H}$ extends to a homeomorphism from $\overline{\mathbb{H}} \cup\{\infty\}$ onto $\overline{\mathbb{H}} \cup\{\infty\}$ we obtain that $F^{-1}(\infty)$ is compact in $\partial D_{1}$. Theorem 1 is then given by Proposition 1.4 condition (ii).

\section{References}

[1] E. BedFord ANd S. Bell, Boundary behavior of proper holomorphic correspondences, Math. Ann. 272(4) (1985), 505-518.

[2] E. Bedford And J. E. Forness, A construction of peak functions on weakly pseudoconvex domains, Ann. of Math. (2) 107(3) (1978), $555-568$.

[3] E. Bedford and S. I. Pinchuk, Convex domains with noncompact groups of automorphisms, Mat. Sb. 185(5) (1994), 3-26.

[4] S. Bell and D. Catlin, Regularity of CR mappings, Math. Z. 199(3) (1988), 357-368.

[5] F. Berteloot, Characterization of models in $\mathbf{C}^{2}$ by their automorphism groups, Internat. J. Math. 5(5) (1994), 619-634.

[6] E. Cartan, Sur l'équivalence pseudo-conforme des hypersurfaces de l'espace de deux variables complexes, Ann. of Math. 11 (1932), $17-90$.

[7] D.-C. Chang and S. Grellier, Régularité de la projection de Szegő dans les domaines découplés de type fini de $\mathbb{C}^{n}, C$. R. Acad. Sci. Paris Sér. I Math. 315(13) (1992), 1365-1370.

[8] B. Coupet, H. Gaussier and A. B. Sukhov, Regularity of CR maps between convex hypersurfaces of finite type, Proc. Amer. Math. Soc. 127(11) (1999), 3191-3200. 
[9] B. Coupet, S. I. Pinchuk and A. B. Sukhov, On boundary rigidity and regularity of holomorphic mappings, Internat. J. Math. 7(5) (1996), 617-643.

[10] B. Coupet And A. B. Sukhov, On CR mappings between pseudoconvex hypersurfaces of finite type in $\mathbf{C}^{2}$, Duke Math. J. 88(2) (1997), 281-304.

[11] B. Coupet And A. B. Sukhov, Rigidity of algebraic CR structures and regularity of CR mappings, Preprint.

[12] J. P. D'Angelo, Finite type conditions for real hypersurfaces, J. Differential Geom. 14(1) (1979), 59-66.

[13] J. E. Fornæss And N. Sibony, Construction of P.S.H. functions on weakly pseudoconvex domains, Duke Math. J. 58(3) (1989), 633-655.

[14] J. E. FORnæSS AND J. D. MCNEAL, A construction of peak functions on some finite type domains, Amer. J. Math. 116(3) (1994), $737-755$.

[15] A. V. Isaev, Rigid spherical hypersurfaces, Complex Variables Theory Appl. 31(2) (1996), 143-163.

[16] J. D. MCNeAL, Local geometry of decoupled pseudoconvex domains, in "Complex analysis" (Wuppertal, 1991), Vieweg, Braunschweig, 1991, pp. 223-230.

[17] S. I. Pinchuk and S. I. Tsyganov, Smoothness of CR-mappings between strictly pseudoconvex hypersurfaces, Izv. Akad. Nauk SSSR Ser. Mat. 53(5) (1989), 1120-1129, 1136.

[18] A. B. SukHov, On the boundary regularity of holomorphic mappings, Mat. Sb. 185(12) (1994), 131-142.

[19] S. M. Webster, On the mapping problem for algebraic real hypersurfaces, Invent. Math. 43(1) (1977), 53-68.

C.M.I.

39 rue Joliot-Curie

13453 Marseille Cedex 13

France

E-mail address: gaussier@cmi.univ-mrs.fr 